\title{
ACCORDANCE: A Novel OFDMA-PON Paradigm for Ultra- High Capacity Converged Wireline-Wireless Access Networks
}

\author{
Konstantinos Kanonakis ${ }^{1}$, Ioannis Tomkos ${ }^{1}$, Thomas Pfeiffer ${ }^{2}$, Josep Prat ${ }^{3}$, Pandelis Kourtessis ${ }^{4}$ \\ ${ }^{I}$ Athens Information Technology Center, 0.8km Markopoulou Ave., 19002 Athens, Greece. \\ Tel: +302106682793,e-mail: kkan@ait.edu.gr \\ ${ }^{2}$ Alcatel-Lucent, Bell Labs Germany, Lorenzstrasse 10, D-70435 Stuttgart, Germany. \\ ${ }^{3}$ Universitat Politècnica de Catalunya, Jordi Girona D4, 08034 Barcelona, Spain. \\ ${ }^{4}$ University of Hertfordshire, College Lane Campus, Hatfield AL10 9AB, UK.
}

\begin{abstract}
This paper presents ACCORDANCE, a novel ultra-high capacity extended reach optical access network architecture based on OFDMA technology/protocols, implemented through the proper mix of state-of-the-art photonics and electronics. Such architecture is not only intended to offer improved performance compared to evolving TDMA-PON solutions but also provide the opportunity for convergence between optical, radio and copper-based access. Although OFDM has been used in radio and copper-based communications, it is only recently that is making its way into optics and is expected to increase the system reach and transmission rates without increasing the required cost/complexity of optoelectronic components. ACCORDANCE hence aims to realize the concept of introducing OFDMA-based technology and protocols (Physical and MAC layer) to provide a variety of desirable characteristics, such as increased aggregate bandwidth and scalability, enhanced resource allocation flexibility, longer reach, lower equipment cost/complexity and lower power consumption, while also supporting multi-wavelength operation. In addition, it enables the convergence of the optical infrastructure with standard wireless solutions, thus offering a way to integrate dominant wired and wireless technologies in a hybrid access network supporting seamless ubiquitous broadband services.
\end{abstract}

Keywords: Passive Optical Network (PON), Orthogonal Frequency Division Multiplexing (OFDM), Orthogonal Frequency Division Multiple Access (OFDMA), OFDM-PON, OFDMA-PON, Converged Access Network.

\section{INTRODUCTION}

Passive optical networks (PONs) based primarily on time division multiple access (TDMA), have evolved as an access solution that provides simplicity and low operational cost with tens of Mbps offered to each user. Nevertheless, the increasing demands of modern applications such as HDTV, 3DTV and ultra-high speed browsing, as well as emerging applications such as multimedia conferencing and multiplayer online gaming have lately lead the research towards increased-capacity optical access solutions. For example, wavelength division multiplexed PONs (WDM-PONs) which enable service providers to offer dedicated wavelengths straight to homes and businesses over the existing optical backbone have been increasingly considered. However, WDMPONs are currently considerably costly to implement, precisely operate and maintain while not offering satisfactory flexibility in terms of bandwidth granularity. Moreover, next generation access networks should ensure that customers receive acceptable QoS whenever or wherever required, whether at a fixed point, such as the home or an office or on-the-go.

Orthogonal Frequency Division Multiplexing (OFDM) technology utilizes several low bit rate sub-carriers of the link to carry different QAM symbols simultaneously and was originally conceived as a modulation method for media displaying bandwidth limitations (copper, radio). When the scheme is employed to allow for Multiple Access (OFDMA), different users are assigned to different OFDM sub-carriers. Recently OFDM(A) has also been making its way into the optics world, both in core [1], and access networks. In the latter case, studies have proven that OFDM can provide high capacity, long-reach and cost-effective operation for PONs [2], [3]. The use of OFDM in optical access networks has mainly been driven by the necessity to increase transmission rates without increasing the bandwidth of optoelectronic transceivers, as opposed to focusing on link optimization.

Instead of solely addressing the physical layer aspects of OFDMA-PONs, the EU FP7 project ACCORDANCE [4] adopts a holistic approach, i.e. one that achieves end-to-end, vertical network connectivity by addressing the both the physical and the Medium Access Control (MAC) layers. The most relevant works to our knowledge are the ones presented in [5] and [6], which however address only a small part of our vision for such a network. The novel OFDMA-based access architecture proposed will be able to provide at the same time a wide variety of desirable characteristics, like increased aggregate bandwidth (even reaching the 100Gbps regime) and scalability, enhanced resource allocation flexibility, longer reach and low CAPEX/OPEX, while being fully compatible with the concept of next generation PONs since it facilitates convergence of the optical infrastructure with standard wireless solutions. Most importantly, this can be essentially achieved over typical splitter-PON infrastructures. 


\section{THE ACCORDANCE ARCHITECTURE}

An example regarding the overall view of the ACCORDANCE architecture is provided in Figure 1. The topology consists of the Optical Line Termination (OLT) located at the Central Office (CO) and several Optical Network Units (ONUs) located either at the user (residential or corporate) premises or at wireless Base Stations (BSs) that are used for providing connectivity to mobile terminals. One single feeder optical fibre from the OLT is used to carry all upstream and downstream signals up to a power splitter which broadcasts them to multiple network segments. Then, further power splitters (for FTTH) are used for broadcasting the signals to all ONUs of each segment. Alternatively, some of those fibres can directly lead to wireless antennas, or switches with DSL interfaces (FTTB case). Therefore, the OLT manages the assignment of traffic to a large number of sub-carriers which travel all along the Optical Distribution Network (ODN), to be demultiplexed only at the user side and vice versa. The sub-carriers are grouped to form FDM channels (with several tens or hundreds of sub-carriers contained within one channel), each of them used for carrying traffic possibly by different providers and employing different technologies (e.g. GPON, EPON, but also wireless or copper-based ones).

Summing up, the Central Office (CO) can generally be thought of as a PON OLT but combining the additional functionalities of a DSLAM or a wireless BS. It employs Frequency Division Multiplexing (FDM) to address different access network segments which, as also shown in Figure 1 can be categorized as follows: (a) OFDMA/DSCA PONs: The term implies network segments where individual sub-carriers of a FDM window are assigned dynamically (Dynamic Sub-Carrier Allocation - DSCA) to different ONUs of the segment, i.e. the multiplexing takes place at the sub-carrier granularity. It is possible though to follow TDMA approaches as well, so that each sub-carrier is shared among ONUs in the time domain. (b) Pure Wireless: In this case a set of subcarriers in a FDM window is dedicated to a group of wireless Base Stations (BS), which actually incorporate the functionality of an ONU as well. Then Radio-over-Fibre (RoF) or Digital-over-Fibre (DoF) techniques can be used to carry the corresponding OFDM wireless signals through the optical infrastructure. (c) Hybrid OpticalWireless: This type of access can be thought of a mixture of cases (a) and (c), whereby optical and wireless users are allowed to coexist and share the sub-carriers within the same FDM window. (d) Legacy or Next-Generation Optical Access: In the case of legacy PON technologies like EPON or GPON, as well as of TDMA-based updates of those protocols, OFDM may only be employed as a modulation (potentially including an RF-todigital converter) rather than multiple access method. (e) Copper-based Access: This is achieved by using FDM windows as transparent pipes for transmitting the corresponding DMT signal up to the DSL users and vice versa.

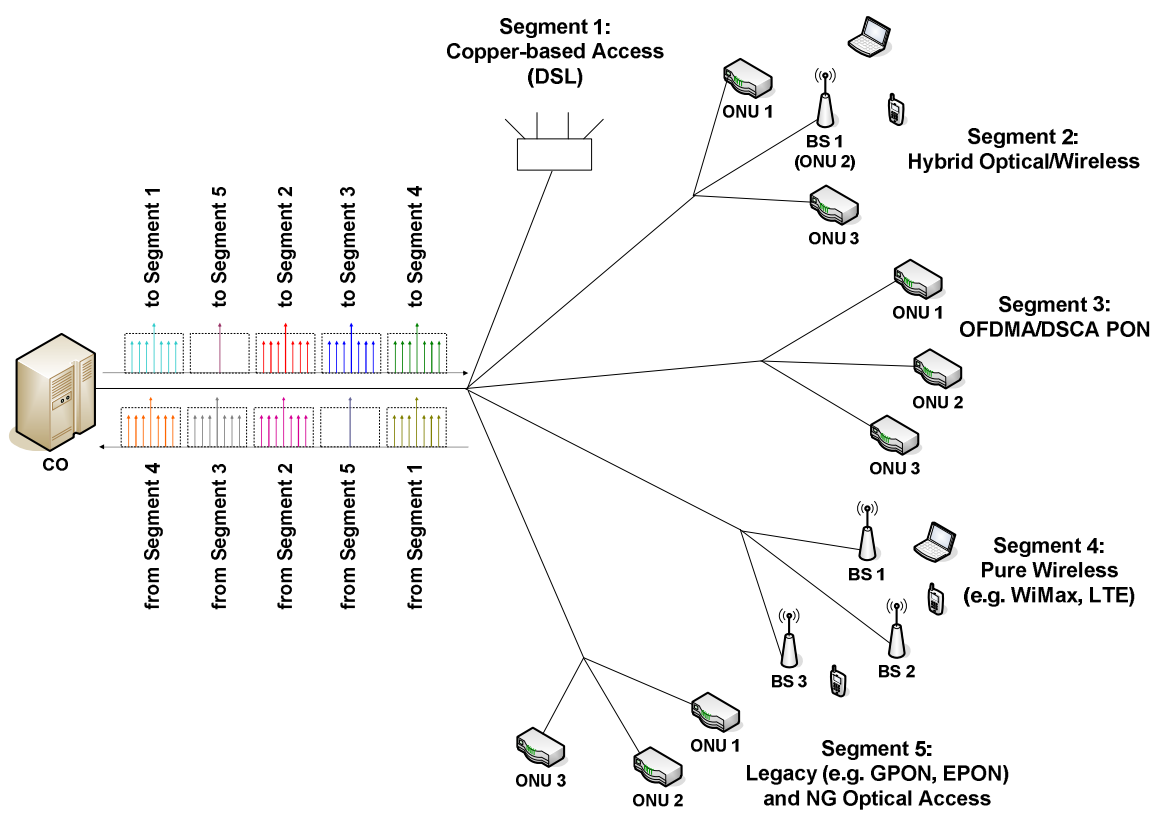

Figure 1: Example of the ACCORDANCE architecture.

\section{NODE ARCHITECTURE AND OPERATION}

For the downstream transmission, a single laser module of very high bandwidth (in the range of 10 to $25 \mathrm{GHz}$ ) is used at the OLT, since the signal is broadcast to all users by means of power splitting. Assuming 16-QAM modulation within all FDM windows, this will lead to aggregate rates ranging from $40 \mathrm{Gbps}$ up to $100 \mathrm{Gbps}$. Although the width of the FDM windows is in the range of few GHz, the bandwidth of each receiver at the user side is the full bandwidth (for example $10 \mathrm{GHz}$ or $25 \mathrm{GHz}$ ) of the laser. The exact receiver design though will 
vary significantly based on the method used for further demultiplexing the spectrum components. There are two main options studied in ACCORDANCE: (a) Optical Field Modulation: By that it is meant that optical field modulation has been performed at the OLT side to produce the downstream signal. At the receiver, optical filtering isolates the required FDM window. Then, only the filtered components need to be further processed electrically. Although this approach imposes many challenges the main benefits are bit rate transparency, lower processing latency and improved energy-efficiency due to the reduced electronic processing effort. (b) Optical Intensity Modulation: The OLT creates the groups of sub-carriers in the RF domain using OFDM-QAM modulation; the produced signal is then modulated onto the optical intensity to come up with the downstream signal. Then the entire optical spectrum must be $\mathrm{O} / \mathrm{E}$ converted at the receiver, while the different FDM windows are extracted in the electrical domain applying e.g. RF filtering and are subsequently electronically processed.

Regardless of the exact method, the selected FDM window is finally downconverted to the electrical domain. Then, Analog-to-Digital converters (ADC) are used to provide the input for further processing in a DSP processing block. The high frequency of operation may be challenging for the ADC (and for DAC) functions, however this may be solved by using an increased number of QAM levels (reducing the bandwidth of each bit stream at the expense of increased SNR requirements). Of course, this will also increase the processing for further demultiplexing and demodulating the sub-carrier streams, [e.g. the FFT and Parallel-to-Serial (P/S) QAM functions].

In the upstream direction, since the data rates for each network segment are lower than the aggregate, lasers with bandwidth in the range of few $\mathrm{GHz}$ will be used at each ONU. At the OLT side, APD or PIN receivers operating in the same bandwidth range can be used after a wavelength demultiplexer in order to receive the signals from each network segment. The upstream direction is extremely challenging, mainly due to the effect of Signal to Signal Optical Beat Interference (SS-OBI), which appears if the sub-carriers used by the various transmitters overlap in the optical frequency domain. There are two main options for combating SS-OBI: (a) The use of WDM for the ONUs, so that they operate sufficiently far from one another. In this case, it would be highly preferable for the ONUs to be colourless in order to keep overall deployment cost low and facilitate network management. (b) The use of hybrid ODFMA/TDMA MAC mechanisms that enforce a strictly synchronized, timeslot-based operation of ONUs. Further options to minimize SS-OBI (e.g. use of low coherence sources like spectrum sliced ones - SLEDs) are investigated within the project.

\section{PHYSICAL LAYER ASPECTS}

The main benefit of OFDM is that each of the sub-carriers transmitted in parallel has a much lower bitrate than the aggregate, making them less susceptible to impairments like chromatic dispersion and E/O band response limitation and distortion. Therefore, aggregate data of several Gbps can travel much longer distances without the need for dispersion compensating modules and with high split ratios [2], [3], while other works have shown that OFDM signals are more robust to Rayleigh backscattered light than NRZ signals used in typical PON architectures [7]. Consequently a larger user base can be covered by each $\mathrm{CO}$ reducing the cost of deployment and maintenance of COs and distant users can easily be covered. In addition, OFDM allows for higher spectral efficiency since the orthogonality of sub-carriers lets them be spaced very close to one another with less interference. Moreover, there is the possibility for flexible adaptive multi-user bit/power allocation per subcarrier in addition to sub-carrier assignment. This allows the system to adapt the modulation format (essentially the QAM levels used) and distribute the power budget to the different ONUs while taking into account the link quality as well as the required data-rate of each of them. Some of the challenges related to the PHY are: (a) An increased peak-to-average power ratio (PAPR), which comes as a results of multiplexing a large number of independent sub-carriers. (b) Non-linearities due to the use of low-speed/low-cost devices (lasers, electronics) by ONUs, so that they are not forced to work at the aggregate rate. (c) High power levels which may be required in the fibre in order to avoid clipping or saturation, and which could potentially cause eye-safety issues. (d) Frequency offset and phase noise sensitivity because of the relatively long symbol length compared to that of the single carrier (especially when higher order constellations are employed).

\section{MAC LAYER ISSUES}

The ACCORDANCE project will propose and evaluate novel MAC protocols and algorithms for dynamic bandwidth assignment of both FDM bands and OFDM sub-carriers with minimal modifications to existing frame formats. For the FDM window assignment, WDM-PON solutions from the literature are can provide the basis for novel solutions tailored for ACCORDANCE. Extra fields will need to be added to the existing EPON/GPON frame formats to be used during registration and bandwidth allocation. Regarding the OFDMA level, any solution must again modify the MAC mechanisms to include spatial in addition to time slot assignment. A first option would be to define a reservation mechanism which reserves sub-carriers for each ONU devoted to it in a circuit-switched fashion [8]. A less rigid approach would divide each sub-carrier to transmission timeslots, so that each ONU could use a sub-carrier only during the timeslots specified by the OLT. An example of this hybrid OFDMA/TDMA way of operation is shown in Figure 2. 
A straightforward solution for optical/wireless MAC interoperation is to assign specific wavelengths or even individual sub-carriers for the wireless transmission and then the OLT and ONUs have to zero those sub-carriers during the OFDM modulation [5]. In other words, the radio channels can be transmitted transparently to BSs through pre-established transmission pipes and extracted at the user side by electrical filters. More complex (both from PHY and MAC points of view) solutions are investigated as well, whereby a large part of the subcarrier bandwidth range is shared among both wireless terminals and static ONUs. Regarding the purely wireless segments of the network, the definition of a novel centralized MAC concept is sought which dynamically allocate bandwidth among BSs efficiently, taking into account their individual temporal needs in unison.

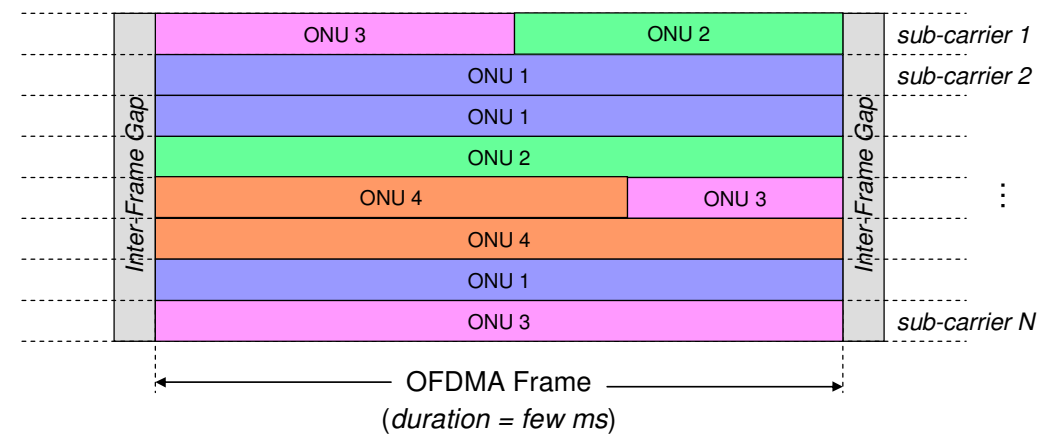

Figure 2: OFDMA/DSCA frame depiction assuming hybrid OFDMA/TDMA operation.

We close with some final comments from a combined PHY and MAC point of view: First of all, note that the PHY and MAC layers can be jointly optimized based on the individual link quality and data-rate requirements for each user, as a result of the QAM-OFDM modulation scheme. In addition, we point out a general trade-off related with the supported number of sub-carriers: A high number of sub-carriers can provide fine granularity with a relatively simple MAC protocol operating only at the sub-carrier domain (each sub-carrier can work at very low rates). This relaxes the strict burst-mode operation requirements of TDMA OLT receivers but implies more challenging PHY aspects (e.g. SS-OBI and non-linearities). On the other hand, fewer sub-carriers operating at higher data rates reduce both PHY challenges and electronic processing. However, in that case TDMA operation is necessary to enable sub-(sub-carrier) allocations.

\section{CONCLUSIONS}

This work has presented the novel OFDMA-PON based architecture studied within the framework of the EU project ACCORDANCE. The expected benefits as well as the challenges regarding all layers of the ACCORDANCE network have been discussed.

\section{ACKNOWLEDGEMENTS}

This work has been partly supported by the E.U. FP7 Project ACCORDANCE.

\section{REFERENCES}

[1] M. Jinno et al., "Spectrum-efficient and scalable elastic optical path network: architecture, benefits, and enabling technologies", IEEE Commun. Mag., Vol. 47, Iss. 11, pp. 66-73, Nov. 2009.

[2] C. W. Chow et al., "Demonstration of High Spectral Efficient OFDM-QAM Long Reach Passive Optical Network", in Proc. European Conference on Optical Communications (ECOC), 2008.

[3] T. N. Duong et al., "10Gbit/s transmission over $2.5 \mathrm{GHz}$ bandwidth by direct modulation of commercial VCSEL and multi-mode FP lasers using Adaptively Modulated Optical OFDM modulation for Passive Optical Network", in Proc. European Conference on Optical Communications (ECOC), 2008.

[4] http://www.ict-accordance.eu/

[5] D. Qian et al., "10-Gb/s OFDMA-PON for Delivery of Heterogeneous Services", in Proc. OFC/NFOEC 2008.

[6] W. Wei et al., "PONIARD: A Programmable Optical Networking Infrastructure for Advanced Research \& Development of Future Internet", J. Lightw. Technol., Vol. 27, No. 3, pp. 233-241, Jan. 2009.

[7] C. W. Chow et al., "Rayleigh Backscattering Performance of OFDM-QAM in Carrier Distributed Passive Optical Networks," IEEE Photonics Technology Letters, Vol. 20, pp. 1848-1850, Nov. 2008.

[8] W. Wei, T. Wang, D. Qian and J. Hu, "MAC Protocols for Optical Orthogonal Frequency Division Multiple Access (OFDMA)-based Passive Optical Networks", in Proc. OFC/NFOEC 2008. 